\title{
Comparação entre as limas \\ Reciproc e WaveOne na formatação e centralização do canal: uma revisão integrativa
}

\author{
Comparison between Reciproc and WaveOne files for shaping and \\ centering the canal: an integrative review
}

\author{
Gabriela Moreira Grossi* \\ Ludmila de Almeida Cunha** \\ Mariane Floriano Lopes Santos Lacerda** \\ Caroline Felipe Magalhães Girelli**** \\ Viviane Ferreira Guimarães Xavier ${ }^{* * * *}$
}

\section{Resumo}

O sucesso do tratamento endodôntico envolve a devida capacidade de limpeza e modelagem dos sistemas de canais radiculares, contudo, não há consenso na literatura sobre os sistemas de lima única Reciproc e WaveOne em termos de formatação do canal. Objetivo: comparar a efetividade na formatação dos canais radiculares utilizando os sistemas Reciproc e WaveOne, no que tange ao transporte e à centralização do preparo. Método: o plano sistemático para a execução desta revisão integrativa consistiu em quatro etapas. Realizou-se um levantamento bibliográfico nas seguintes bases de dados: SciELO, PubMed e Lilacs, com os seguintes descritores: Reciproc e WaveOne, shaping canal, centering canal. Foi feita a leitura dos resumos que constavam nos artigos e documentos selecionados e a seleção daqueles que contemplavam de três a cinco descritores sobre o tema desta pesquisa. Em seguida, foi feita a leitura dos textos na íntegra, seguida pela construção de uma tabela com as informações levantadas nesse processo. Resultados: dos artigos selecionados, sete foram publicados em revistas odontológicas, sendo cinco publicados em revistas de área específica (endodontia) e apenas um foi publicado em uma revista de pesquisa básica de tecnologia. Verificou-se que a maior parte dos estudos foi publicada nos anos 2014 e 2015, a maioria publicada no Brasil e na Coreia. Conclusão: com base nos artigos estudados, não foi possível con- cluir que há diferença entre os instrumentos avaliados, quanto à centralização e à formatação do preparo após a utilização de Reciproc e WaveOne.

Palavras-chave: Endodontia. Tratamento do canal radicular. Equipamentos odontológicos.

\section{Introdução}

O maior desafio na endodontia é a formatação e a desinfecção eficaz dos canais radiculares, a remoção de bactérias e seus subprodutos, resíduos de necrose pulpar e ou dentina contaminada ${ }^{1,2}$, visto que a complexidade anatômica dos canais radiculares impõe limitações durante a construção do canal cirúrgico ${ }^{2,4}$.

Tradicionalmente, o preparo químico-mecânico (PQM) é realizado com limas manuais por meio de diferentes técnicas. No entanto, esse preparo demanda um grande número de instrumentos e, ainda, pode provocar desvios, perfurações e grandes desgastes radiculares, devido à elevada rigidez de tais instrumentos, que são produzidos em liga de aço-inoxidável ${ }^{5,6}$.

Cirurgiã-dentista, aluna do Curso de Especialização em Endodontia - Associação Brasileira de Odontologia/Governador Valadares (ABO/GV ), Governador Valadares, MG, Brasil.

Professora especialista do Curso de Especialização em Endodontia - ABO/GV, Governador Valadares, MG, Brasil.

Professora doutora do Departamento de Odontologia da Universidade Federal de Juiz de Fora - Campus Avançado Governador Valadares (UFJF/GV), Governador Valadares, MG, Brasil.

**** Professora mestre do Curso de Especialização em Endodontia - ABO/GV, Governador Valadares, MG, Brasil.

Professora especialista do Curso de Especialização em Endodontia - ABO/GV, Governador Valadares, MG, Brasil. 
Com a finalidade de promover um melhor PQM, surgiram os instrumentos de níquel-titânio (Ni-Ti), que possibilitam o tratamento de canais radiculares com alto grau de complexidade de maneira mais ágil, eficaz e com menor índice de iatrogenias, proporcionando um canal cirúrgico com características morfológicas próximas ao canal anatômico ${ }^{7}$.

Assim, as características mecânicas e a flexibilidade dessa liga de Ni-Ti permitiram a introdução das limas rotatórias, as quais apresentam diferentes configurações de seção transversal e variação dos diâmetros da ponta ativa e da conicidade. Dessa forma, sequências de instrumentos com design e diâmetros variados passaram a ser utilizados, dando origem ao sistema rotatório, em que cada lima da sequência trabalha em um segmento do canal radicular, executando uma rotação de $360^{\circ}$. A desvantagem do sistema rotatório, no entanto, consiste na necessidade de se utilizar uma série de instrumentos para completar o PQM e, ainda, o risco de fratura desses instrumentos ${ }^{8}$.

Introduzido por Yared, em 2008, o movimento reciprocante foi proposto como uma nova perspectiva às limas de Ni-Ti. Essa cinemática corresponde a dois movimentos mecânicos durante a instrumentação, um para ação de corte no sentido anti-horário, que determina que o instrumento avance no canal e se envolva na dentina para cortá-la, e outro recíproco, que serve para liberação do instrumento no sentido horário, permitindo que a lima seja imediatamente desengatada e progrida de forma segura ao longo do canal, enquanto reduz o efeito de enroscamento e separação da lima ${ }^{9,12}$. Essa técnica aumenta a sobrevida do instrumento, reduzindo o índice de fratura quando comparada à cinemática de rotação contínua.

Um exemplo desses novos instrumentos é o sistema de lima única Reciproc (VDW GmbH, Munique, Alemanha), que possui corte transversal, uma ponta não cortante e lâminas afiadas que moldam o canal por meio de um movimento recíproco com uma velocidade de $300 \mathrm{rpm}\left(150^{\circ}\right.$ no sentido anti-horário e, em seguida, $30^{\circ}$ no sentido horário $)^{9}$. Esse sistema está disponível em três tamanhos e conicidades diferentes; R25 (25/.08), R40 (40/.06) e R50 (50/.05) $)^{7,13-15}$.

Já o sistema de lima única WaveOne (Dentsply-Mailefer, Ballaigues, Suíça) apresenta geometria de um cone inverso, ângulo helicoidal variável e uma borda não ativa; é usado com $170^{\circ}$ no sentido anti-horário e $50^{\circ}$ no sentido horário, a uma velocidade de $300 \mathrm{rpm}$. Está disponível em diferentes tamanhos de ponta e conicidades: 20/06, 25/08 e $40 / 08^{7,16}$.

Ambos os sistemas de instrumentação reciprocante têm a proposta de serem utilizados como instrumento único e de uso único para o preparo do canal radicular, tornando o tratamento mais rápido, diminuindo a fadiga cíclica e eliminando a contaminação cruzada ${ }^{9}$. Além dessa cinemática diferen- ciada, ambos são constituídos de liga de Ni-Ti com tratamento especial, conhecido como M-Wire, que resulta em melhorias do instrumento, como maior: flexibilidade, resistência à flexocompressão e à fratura por torsão, o que propicia uma instrumentação mais centralizada e, consequentemente, melhores resultados ${ }^{17}$.

Sabe-se que o design da seção transversal desses instrumentos minimiza a área de contato entre a lima e a parede dentinária, o que, segundo a literatura, pode reduzir o risco de transporte do canal ${ }^{18}$. Portanto, instrumentos diferentes apresentam tendências diferentes para formatações e centralizações do preparo, devido às suas diferenças nas seções transversais.

Diante do exposto, o presente estudo teve por objetivo comparar a efetividade na formatação dos canais radiculares utilizando os sistemas Reciproc e WaveOne, no que tange ao transporte e à centralização do preparo, por meio de uma revisão integrativa.

\section{Materiais e método}

Este estudo tem uma abordagem qualitativa, em que se optou pelo método da revisão integrativa para alcance do objetivo proposto ${ }^{19,20}$. Esta revisão integrativa da literatura compara as limas únicas Reciproc e WaveOne na formatação e na centralização do canal. A revisão integrativa permite a incorporação das evidências na prática clínica ${ }^{21}$.

De acordo com as etapas de elaboração de uma revisão integrativa, o primeiro passo foi determinar um objetivo para nortear a busca na literatura. Assim, a questão que embasou a coleta de evidências científicas deste estudo foi: qual das limas é mais eficaz no tratamento endodôntico sem causar deformidades e desvios no canal radicular, Reciproc ou WaveOne?

As bases de dados on-line utilizadas para a revisão da literatura foram: PubMed (base de dados desenvolvida pela National Center for Biotecnology Information na National Library of Medicine), SciELO (Biblioteca Eletrônica de Periódicos Científicos Brasileiros) e Lilacs (Literatura Latino-Americana em Ciências da Saúde). Os descritores utilizados no levantamento da literatura foram: Reciproc e WaveOne, shaping canal, centering canal.

Os critérios de elegibilidade deste estudo foram: os artigos estarem disponíveis na íntegra nas bases de dados pesquisadas, serem publicados entre novembro de 2012 e novembro de 2016 e estarem no idioma inglês. Tais artigos deveriam abordar a comparação das limas Reciproc e WaveOne na formatação e na centralização do canal. Foram descartados estudos que não compararam as respectivas limas, bem como artigos em outras línguas que não a inglesa, artigos que não tinham resumo e estudos que utilizaram dentes decíduos. Para verificar a compatibilidade dos artigos com o objetivo do estudo, 
as análises foram realizadas por revisores independentes e, posteriormente, comparadas.

Após a leitura dos artigos selecionados, as informações foram coletadas e digitadas em banco de dados de acordo com as seguintes variáveis: Autor/ Ano, Objetivo, Metodologia e Conclusão. Todos os registros foram armazenados em um arquivo específico do programa Microsoft Excel 2007.

\section{Resultados}

A revisão de literatura foi realizada após a conferência dos artigos na íntegra e dos critérios de elegibilidade pré-estabelecidos. Dentre os artigos pesquisados, todos foram publicados em periódicos de procedência internacional. Ao total, foram encontrados 999 artigos. Em relação às bases de dados, verificaram-se os seguintes resultados: na PubMed, foram encontrados 876 artigos, sendo incluídos 6; na SciELO, 39 artigos, sendo 2 incluídos; na Lilacs, 84 artigos, porém nenhum foi selecionado para a revisão integrativa. Após a remoção de duplicações nas bases de dados consultadas, oito artigos foram incluídos neste estudo (Quadro 1).

Dos artigos selecionados, sete foram publicados em revistas odontológicas, sendo cinco publicados em revistas de área específica (endodontia), e apenas um foi publicado em uma revista de pesquisa básica de tecnologia. Verificou-se que a maior parte dos estudos foi publicada nos anos 2014 e 2015 e no Brasil e na Coreia.

Quadro 1 - Identificação dos estudos selecionados para revisão integrativa

\begin{tabular}{|c|c|c|c|}
\hline Autores/Ano & Objetivo & Metodologia & Conclusão \\
\hline $\begin{array}{l}\text { Dhingra et al. }{ }^{22} \\
(2015)\end{array}$ & $\begin{array}{l}\text { Comparar e avaliar os sistemas WaveO- } \\
\text { ne, Reciproc e OneShape em transporte } \\
\text { do canal, espessura da dentina cervical } \\
\text { e área seccional transversa nos primeiros } \\
\text { molares inferiores, usando tomografia } \\
\text { computadorizada. }\end{array}$ & $\begin{array}{l}\text { Foram avaliados, antes e após a ins- } \\
\text { trumentação, o transporte do canal, } \\
\text { a espessura da dentina cervical e a } \\
\text { área transversal, e as imagens foram } \\
\text { sobrepostas por um software CS3D. }\end{array}$ & $\begin{array}{l}\text { Não houve diferença significativa } \\
\text { entre WaveOne e Reciproc. }\end{array}$ \\
\hline $\begin{array}{l}\text { Lim et al. }{ }^{23} \\
(2013)\end{array}$ & $\begin{array}{l}\text { Avaliar a capacidade de limas WaveOne } \\
\text { primária e Reciproc R25 em manter o ca- } \\
\text { nal original e sua curvatura com ou sem } \\
\text { pré-alargamento. }\end{array}$ & $\begin{array}{l}\text { Foi avaliada a capacidade de centra- } \\
\text { lização e desvio de cada grupo em } 1 \text {, } \\
2,3,5 \text { e } 7 \text { mm. Imagens pré e pós- } \\
\text {-instrumentação foram sobrepostas } \\
\text { utilizando um software Photoshop. }\end{array}$ & $\begin{array}{l}\text { Nos níveis de } 1 \mathrm{~mm} \text { e } 2 \mathrm{~mm} \text {, hou- } \\
\text { ve maior centralização para Wa- } \\
\text { veOne. Nos demais, a centraliza- } \\
\text { ção foi semelhante em ambos. }\end{array}$ \\
\hline $\begin{array}{l}\text { Silva et al. }{ }^{24} \\
(2015)\end{array}$ & $\begin{array}{l}\text { Avaliar a capacidade de limas únicas } \\
\text { ProTaper Universal, Reciproc, WaveOne } \\
\text { e ProTaperNext na manutenção da ana- } \\
\text { tomia original do canal radicular. }\end{array}$ & $\begin{array}{l}\text { Blocos de resina foram fotografados } \\
\text { pré e pós-instrumentação em por- } \\
\text { ções retas e curvas e foram avaliados } \\
\text { o transporte e a centralização do ca- } \\
\text { nal. }\end{array}$ & $\begin{array}{l}\text { WaveOne produziu maior trans- } \\
\text { porte do canal, enquanto Reci- } \\
\text { proc exibiu maior centralização } \\
\text { do preparo. }\end{array}$ \\
\hline $\begin{array}{l}\text { Netto et al. }{ }^{25} \\
(2014)\end{array}$ & $\begin{array}{l}\text { Avaliar alterações anatômicas no terço } \\
\text { médio de incisivos inferiores após utili- } \\
\text { zação das limas Reciproc, WaveOne e } \\
\text { Tilos. }\end{array}$ & $\begin{array}{l}\text { Pré e pós-instrumentação utilizando } \\
\text { tomografia computadorizada para } \\
\text { avaliar centralização e desvio do ca- } \\
\text { nal radicular. }\end{array}$ & $\begin{array}{l}\text { Reciproc apresentou menor des- } \\
\text { vio, maior centralização e melhor } \\
\text { formatação do canal do que Wa- } \\
\text { veOne. }\end{array}$ \\
\hline $\begin{array}{l}\text { Burklein et al. }{ }^{26} \\
(2014)\end{array}$ & $\begin{array}{l}\text { Comparar a habilidade de formatação de } \\
\text { diferentes sistemas rotatórios e recipro- } \\
\text { cantes com ou sem pré-alargamento em } \\
\text { canais curvos. }\end{array}$ & $\begin{array}{l}\text { Blocos de resina foram avaliados } \\
\text { pré e pós-instrumentação. Imagens } \\
\text { foram sobrepostas por um software. }\end{array}$ & $\begin{array}{l}\text { WaveOne teve maior deformida- } \\
\text { de no canal que Reciproc. }\end{array}$ \\
\hline $\begin{array}{l}\text { Gergi et al. }{ }^{27} \\
(2014)\end{array}$ & $\begin{array}{l}\text { Avaliar e comparar o transporte de canais } \\
\text { radiculares curvos usando Reciproc, Wa- } \\
\text { veOne e Twist File Adaptive (TF). }\end{array}$ & $\begin{array}{l}\text { Foram registradas imagens pré e } \\
\text { pós-instrumentação por tomografia } \\
\text { computadorizada, quanto ao grau de } \\
\text { transporte do canal e centralização. }\end{array}$ & $\begin{array}{l}\text { Reciproc possui maior grau de } \\
\text { transporte do canal. WaveOne } \\
\text { tem melhor centralização e menor } \\
\text { eficiência de corte do que Reci- } \\
\text { proc. }\end{array}$ \\
\hline $\begin{array}{l}\text { Bane et al. }{ }^{28} \\
(2015)\end{array}$ & $\begin{array}{l}\text { Avaliar e comparar a capacidade de for- } \\
\text { matação das limas WaveOne, Reciproc } \\
\text { e ProTaper em canais curvos de molares } \\
\text { inferiores humanos extraídos. }\end{array}$ & $\begin{array}{l}\text { Foram feitas radiografias pré e pós- } \\
\text {-instrumentação e as medições foram } \\
\text { realizadas usando o software Adobe } \\
\text { Photoshop CS3. }\end{array}$ & $\begin{array}{l}\text { Reciproc é melhor na formatação } \\
\text { de canais severamente curvos e } \\
\text { causa menores desvios e fraturas } \\
\text { que WaveOne. }\end{array}$ \\
\hline $\begin{array}{l}\text { Yoo e } \mathrm{Cho}^{29} \\
(2012)\end{array}$ & $\begin{array}{l}\text { Comparar a capacidade de formatação } \\
\text { dos sistemas Reciproc, WaveOne, ProTa- } \\
\text { per, Profile e Limas K manuais em canais } \\
\text { simulados. }\end{array}$ & $\begin{array}{l}\text { Imagens pré e pós-instrumentação } \\
\text { foram tiradas em microscópio e foi } \\
\text { medida a quantidade de resina re- } \\
\text { movida. }\end{array}$ & $\begin{array}{l}\text { Reciproc e WaveOne mantêm } \\
\text { melhor formatação do canal pre- } \\
\text { servando sua anatomia original. }\end{array}$ \\
\hline
\end{tabular}

Fonte: dados da pesquisa. 


\section{Discussão}

Com a utilização da liga de níquel-titânio (Ni-Ti) para fabricação das limas endodônticas, principalmente para as acionadas em motores, foi possível driblar dificuldades anatômicas dos sistemas de canais radiculares, o que não era possível com o uso de limas de aço inoxidável, que dificultava o sucesso do tratamento endodôntico ${ }^{30}$.

Diferentemente dos motores elétricos da primeira geração, os mais atuais possuem controle de torque e velocidade, bem como o antirreverso. Alguns possuem tanto o movimento rotatório contínuo como o reciprocante, além de controle de angulação para avanço e recuo do instrumento quando acionado em movimento reciprocante. Assim, com os avanços nos motores, os fabricantes iniciaram modificações na estrutura das limas, na composição da liga de Ni-Ti e na técnica de fabricação, a fim de melhorar o desempenho dos instrumentos e minimizar as iatrogenias durante o $\mathrm{PQM}^{31}$.

Alguns estudos ${ }^{32,33}$ mostraram que a resistência à fratura cíclica dos instrumentos aumenta quando utilizados no movimento reciprocante, além disso, observou-se que o uso de limas reciprocantes proporciona preparos mais conservadores quando comparados aos preparos com o sistema rotatório. Tal fato pode ser justificado pela utilização de apenas um instrumento para conformação do canal radicular com o sistema reciprocante, enquanto, no sistema rotatório, utiliza-se uma sequência de limas ${ }^{34,38}$.

A presença de preparos mais centralizados tem sido proporcionada com o advento de manejos térmicos na liga de $\mathrm{Ni}$ - $\mathrm{Ti}$, os quais permitem o desenvolvimento de instrumentos com composições cristalinas alteradas, possibilitando estágios intermediários entre as fases austenítica e martensítica, que, consequentemente, passam a apresentar comportamentos diferentes, como maior resistência à fadiga cíclica e menor transporte de canais ${ }^{39}$.

Recentemente, novas limas têm sido submetidas ao tratamento térmico denominado M-Wire, responsável por aumentar a flexibilidade dos instrumentos e, com isso, evitar o excesso de remoção dentinária nas zonas críticas, como os terços médios de raízes achatadas de incisivos e pré-molares inferiores e as raízes mesiais de molares inferiores, bem como desvios foraminais, com consequentes perfurações radiculares ${ }^{24}$.

Os sistemas WaveOne e Reciproc utilizam movimentos oscilatórios assimétricos (sentidos horário/ anti-horário), contudo, com grau de oscilação diferentes para cada sistema, diferentes conicidades e diferentes geometrias da seção transversal ${ }^{27}$. Esses fatores levam tais instrumentos a apresentarem diferenças entre eles, tanto na resistência à fadiga como ao torque ${ }^{17}$, fato que justifica esta revisão integrativa sobre a comparação da formatação e da centralização do canal dos instrumentos WaveOne e Reciproc.
As limas WaveOne podem ser usadas em qualquer canal, independentemente do seu comprimento, diâmetro ou curvatura. Esse sistema utiliza ângulos desiguais, que são quatro vezes mais seguros e três vezes mais rápidos do que os instrumentos manuais. Além disso, o instrumento tem hélice reversa e duas seções transversais distintas sobre o comprimento com uma seção convexa triangular modificada, permitindo uma boa centralização do canal sem causar deformidades e desvios ${ }^{26,40}$. Já as limas Reciproc apresentam uma ação de corte muito maior do que o desgaste, permitindo uma melhor progressão apical e uma eficiência mais elevada. Além disso, são consideradas ideais para canais extremamente estreitos e invisíveis em radiografias, como os quartos canais de primeiros molares superiores ${ }^{27}$

Em relação ao transporte apical, diversos estu$\operatorname{dos}^{22,27,29,41}$ demonstraram que não houve diferença significante entre WaveOne e Reciproc. Uma das razões refere-se ao fato de os instrumentos apresentarem pontas não cortantes que trabalham com pressão mínima apical e funcionam apenas como guia para uma fácil penetração no canal, dificultando o transporte apical/desvio. Entretanto, outros trabalhos ${ }^{24,25}$ verificaram que Reciproc provocou menor desvio apical. A variação dos resultados pode ser explicada devido às diferenças metodológicas dos estudos, tais como a diversidade da amostra, com o uso de blocos de resina ${ }^{24}$ ou de dentes humanos, ${ }^{22,25,27,29} \mathrm{e}$ da avaliação pré e pós-instrumentação.

Quanto à centralização do preparo, observou-se que não houve diferença significativa entre Reciproc e WaveOne ${ }^{27,29}$. No entanto, alguns trabalhos ${ }^{24,25}$ verificaram que Reciproc apresentou melhor centralização do preparo quando comparado com WaveOne.

No que diz respeito à espessura da dentina cervical, resultados ${ }^{22}$ verificaram que não houve diferença estatística entre os sistemas WaveOne e Reciproc. No entanto, outros estudos ${ }^{25,27,42,44}$ demonstraram que as limas Reciproc promoveram menor remoção dentinária e mantiveram melhor a anatomia interna original do canal do que limas WaveOne.

$\mathrm{Na}$ qualidade do preparo radicular, observou-se melhor formatação do canal radicular pela lima Reciproc quando comparada ao sistema WaveO$\mathrm{ne}^{25,26,28}$. Esse resultado, provavelmente, deve-se ao fato de a Reciproc possuir menor conicidade e número de espiras que WaveOne e pelo ângulo de corte ser levemente positivo em função da sua seção transversal em "S"

A ausência de unanimidade nos resultados dos estudos pode ser associada aos variados materiais e técnicas utilizados nos diferentes estudos. A determinação do transporte apical e da centralização do preparo do canal radicular foi baseada em um modelo previamente descrito ${ }^{46}$. Desde então, vários estudos aplicaram diferentes metodologias para essa avaliação, como uso de canais artificias ${ }^{47,48}$ ou de dentes humanos. A avaliação pré e pós-instru- 
mentação também difere entre os estudos, com a utilização de imagens radiográficas periapicais ${ }^{49}$, microscopia eletrônica de varredura ${ }^{50} \mathrm{e}$, mais recentemente, por meio de tomografia computadorizada de feixe cônico ${ }^{27,41}$, além de por microtomografia computadorizada ${ }^{27,51-53}$.

\section{Conclusão}

As diferenças metodológicas entre os estudos avaliados impuseram limitações às avaliações entre os dois sistemas estudados, com isso, não é possível concluir que há diferença entre os instrumentos avaliados quanto à centralização e à formatação do preparo.

\section{Abstract}

The success of endodontic treatment involves the cleaning and modeling ability of root canal systems, but there is no consensus in the literature on the Reciproc and WaveOne single file systems in terms of canal shaping. Objective: to compare the effectiveness of root canal shaping using Reciproc and WaveOne systems regarding transportation and centralization of the preparation. Method: the systematic plan for conducting this integrative review consisted of four stages. A bibliographic survey was carried out in the following databases: SCIELO, PubMed, and Lilacs, with the following descriptors: Reciproc and WaveOne, shaping canal, centering canal. The abstracts included in the articles and documents selected were read and those including three to five descriptors on the subject of this research were selected. Then, the full texts were read, followed by the construction of a table with information gathered in this process. Results: from the articles selected, seven were published in dental journals, five of which were published in area-specific journals (endodontics), and only one was published in a basic technology research journal. It was verified that most of the studies were published in the years 2014 and 2015, in Brazil and Korea. Conclusion: based on the articles studied, it was not possible to perceive difference between the evaluated instruments regarding centering and shaping of the preparation after the use of Reciproc and WaveOne systems.

Keywords: Endodontics. Root canal treatment. Dental instruments.

\section{Referências}

1. Taşdemir T, Aydemir H, Inan U, Unal O. Canal preparation with Hero 642 rotary Ni-Ti instruments compared with stainless steel hand k-file assessed using computed tomography. Int Endod J 2005; 38(6):402-8.

2. Mauger MJ, Schindler WG, Walker WA. An evaluation of canal morphology at different levels of root resection in mandibular incisor. J Endod 1998; 24(9):607-9.

3. Marchesan MA, Arruda MP, Silva-Sousa YT, Saquy PC, Pecora JD, Sousa-Neto MD. Morphometrical analysis of cleaning capacity using niquel-titanium rotary instrumentation associated with irrigating solutions in mesio-distal flattened root canals. J Appl Oral Sci 2003; 11(1):55-9.
4. Loizides A, Eliopoulos D, Kontakiotis E. Root canal transportation with a Ni-Ti rotary file system and stainless steel hand files in simulated root canals. Quintessence Int 2006; 37(5):369-74.

5. Peters AO. Current challenges and concepts in the preparation of root canal systems: a review. J Endod 2004; 30:559-67.

6. Chen JL, Messer HH. A comparison of stainless steel hand and rotary nickel-titanium instrumentation using a silicone impression technique. Aust Dent J 2002; 47(1):12-20.

7. Johnson E, Lloyd A, Kuttler S, Namerow K. Comparison between a novel nickel-titanium alloy and 508 nitinol on the cyclic fatigue life of ProFile 25/.04 rotary instruments. J En$\operatorname{dod} 2008$; 34(11):1406-9.

8. Marceliano-Alves, MFV, Sousa-Neto MD, Fidel SR, Steier L, Robinson JP, Pécora JD, et al. Shaping ability of single-file reciprocating and heat-treated multifile rotary systems: a micro-CT study. Int Endod J 2014; 48(12):1129-36.

9. Yared G. Canal preparation using only one Ni-Ti rotary instrument: preliminary observations. Int Endod J 2008; 41(4):339-44.

10. Berutti E, Chiandussi G, Paolino DS, Scotti N, Cantatore G, Castellucci A, et al. Canal shaping with WaveOne Primary reciprocating files and ProTaper system: a comparative study. J Endod 2012; 38(4):505-9.

11. De-Deus G, Brandao MC, Barino B, Giorgi KD, Feidl RASF, Luna AS. Assessment of apically extruded debris produced by the single-file ProTaper F2 technique under reciprocating movement. Oral Surg Oral Med Oral Pathoy Oral Radiol Endod 2010; 110(3):390-4.

12. Varela-Patiño P, Ibañes-Párraga A, Rivas-Mundiña B, Cantarore G, Otero XL, Martin-Biedma B. Alternating versus continuous rotation: a comparative study of the effect on instrument life. J Endod 2010; 36(1):157-9.

13. VDW-Munich. [Internet]. Munich, Germany [cited 2015 March 3]. Available from URL: http://www.vdw-dental.com/ en/service/information-and-downloads/catalogues-and-brochures.htm.

14. Kim HC, Kwak SW, Cheung GS, Ko DH, Chung SM, Lee W. Cyclic fatigue and torsional resistance of two new nickeltitanium instruments used in reciprocation motion: Reciproc versus WaveOne. J Endod 2012; 38(4):541-4.

15. Nazari MK, Shahab S, Rostami G. Canal transportation and centering ability of twisted file and reciproc: a cone-beam computed tomography assessment. Iran Endod J 2014; 9(3):174-9.

16. Burklein S, Hinschitza K, Dammaschke T, Schafer E. Shaping ability and cleaning effectiveness of two single-file systems in severely curved root canals of extracted teeth: Reciproc and WaveOne versus Mtwo and ProTaper. Int Endod J 2012; 45(5):449-61

17. Plotino G, Grande NM, Testarelli L, Gambarini G. Cyclic fatigue of Reciproc and WaveOne reciprocating instruments. Int Endod J 2012; 45:614-8.

18. Alapati SB, Brantley WA, Iijima M, Clark WAT, Kovarik L, Buie C, et al. Metallurgical characterization of a new nickel-titanium wire for rotary endodontic instruments. J Endod 2009; 35:1589-93.

19. Benefield LE. Implementing evidence-based practice in home care. Home Health Nurse 2003; 21(12):804-11.

20. Polit DF, Beck CT. Using research in evidence-based nursing practice. In: Polit DF, Beck CT, editors. Essentials of nursing research. Methods, appraisal and utilization. Philadelphia: Lippincott Williams \& Wilkins; 2006. p. 457-94.

21. Roman AR, Friedlander MR. Revisão integrativa de pesquisa aplicada à enfermagem. Cogitare Enferm 1998; 3(2):109-12. 
22. Dhingra A, Ruhal N, Miglani A. Evaluation of Single File Systems Reciproc, Oneshape, and WaveOne using Cone Beam Computed Tomography - an in vitro study. J Clin Diagn Res 2015; 9(4):30-4.

23. Lim YJ, Park SJ, Kim HC, Min KS.. Comparasion of the centering ability of Waveone and Reciproc nickel-titanium in simulated curved canals. Restor Dent Endod 2013; 38(1):21-5.

24. Silva EJ, Vieira VC, Tameirão MD, Belladonna FG, Neves A de A, Souza EM, et al. Quantitative transportation assessment in curved canals prepared with an off-centered rectangular design system. Braz Oral Res 2016; 30(1):e43.

25. Netto C, Palo RM, Pinto LF, Mello-Moura ACV, Daltoé G, Wilhelmsen NSW. CT study of the performance of reciprocating and oscillatory motions in flattened root canal areas. Braz Oral Res 2014; 29(1):1-6.

26. Burklein S, Poschmann T, Schafer E. Shaping ability of different nickel-titanium systems in simulates S-shaped canals with and without glide path. J Endod 2014; 40(8):1231-4.

27. Gergi R, Arbab-Chirani R, Osta N, Naaman A. Micro-computed tomographic evaluation of canal transportation instrumented by different kinematics rotary nickel-titanium instruments. J Endod 2014; 40(8):1223-7.

28. Bane K, Faye B, Sarr M, Niang SO, Ndiaye D, Machtou P. Root canal shaping by single-file systems and rotary instruments: a laboratory study. Iran Endod J 2015; 10(2):135-9.

29. Yoo YS, Cho YB. A comparison of the shaping ability of reciprocating NiTi instruments in simulated curved canals. Restor Dent Endod 2012; 37(4):220-7.

30. Kurnet GG, Camargo Fontanella VR, de Moura AA, Barletta FB. Analysis of apical root transportation associated with Protaper universal F3 and F4 instruments in artificial canals. J Endod 2010; 36(6):1052-5.

31. Pruett JP, Clement DJ, Carnes DLJ. Cyclic fatigue testing of nickel-titaniun endodontic instruments. J Endod 1997; 23(2):77-85.

32. Pérez-Higueras JJ, Arias A, de la Macorra JC. Fadigue resistance of K3, K3XF, and Twisted file Nickel-titanium files under continuous rotation or reciprocating motion. J Endod 2013; 39(12):1585-8

33. Karatas E, Gunduz H, Kirici D, Arslan H, Topcu MC, Yeter Ky. Dentinal crack formation during root canal preparation by the Twisted file adaptive, Protaper next, Protaper Universal and Waveone instruments. J Endod 2015; 41(2):261-4.

34. Hwang YH, Bae KS, Baek SH, Kum KY, Lee W, Shon WJ, et al. Shaping ability of the conventional nickel-titanium and reciprocating nickel-titanium file systems: A comparative study using Micro-computed tomography. J Endod 2015; 40(8):1186-9.

35. Burklein S, Hinschitza K, Dammaschke T, Schafer E. Shaping ability and cleaning effectiveness of two single-file systems in severely curved root canals of extracted teeth: Reciproc and WaveOne versus Mtwo and ProTaper. Int Endod J 2012; 45(5):449-61.

36. Gambarini G, Gergi R, Naaman A, Osta N, Al Sudani D. Cyclic fatigue analysis of twisted file rotary NiTi instruments used in reciprocating motion. Int Endod J 2012; 45(9):802-6.

37. Higuera O, Plotino G, Tocci L, Carrillo G, Gambarini G, Jaramillo DE, et al. Cyclic fadigue resistance of 3 different Nickel-titanium reciprocating instruments in artificial canals. J Endod 2015; 41(6):913-5.

38. Capar ID, Ertas H, Arslan H. Comparison of cyclic fatigue resistance of novel nickel-titanium rotary instruments. Aust Endod J 2015; 41(1):24-8.

39. Young-Jun L, Su-Jung P, Hyeon-Cheol K, Kyung-San M. Comparison of the centering ability of Wave-One and Reciproc nickel-titanium instruments in simulated curved canals. Restor Dent Endod 2013; 38(1):21-5.
40. Annil D, Nidhi R, Anjali M. Evaluation of single file systems Reciproc, Oneshape, and WaveOne using cone beam computed tomography - an in vitro study. J Clinic Diagn Res 2015; 9(4):ZC30-ZC34.

41. Young-Sil Y, Yong-Bum C. A comparison of the shaping ability of reciprocating NiTi instruments in simulated curved canals. Restor Dent Endod 2012; 4(11):220-7.

42. Kuhn G, Jordan L. Fadigue and mechanical properties of nickel-titanium endodontics instruments. J Endod 2002; 28:716-20.

43. Silva EJNL, Vieira VCG, Tameirão MDN, Belladonna FG, Neves AA, Souza EM, et al. Quantitative transportation assessment in curved canals prepared with an off-centered rectangular design system. Braz Oral Res 2016; 30(1):1-7.

44. Saleh AM, Gilani PV, Tavanafar S, Schafer E. Shaping ability of 4 different single-file systems in simulated S-shaped canals. J Endod 2015; 41(4):548-52

45. Versiani MA, Leoni GB, Steier L, De-Deus G, Tassani S Pécora JD, et al. Micro-computed tomography study of oval-shaped canals prepared with the self-adjusting file, Reciproc, WaveOne, and ProTaper universal systems. J Endod 2013; 39(8):1060-6.

46. Gambill JM, Alder M, del Rio CE. Comparison of nickel-titanium and stainless steel hand-file instrumentation using computed tomography. J Endod 1996; 22(7):369-75.

47. Wu H, Peng C, Bay Y, Hu X, Wang L, Li C. Shaping ability of Protaper Universal, WaveOne and protaper Next in simulated L-shaper and S-shaped root canals. BMC Oral Health $2015 ; 1: 15-27$.

48. Liu W, Wu B. Root canal surface strain and canal center transportation induced by 3 different nickel-titanium rotary instruments systems. J Endod 2016; 42:299-303.

49. Burkelin S, Mathey D, Schafer E. Shaping ability of ProTaper Next and BT-Race nickel-titanium instruments in severely curved root canals. Int Endod J 2015; 48(8):774-81.

50. Schafer E, Vlassis M. Comparative investigation of two rotary nickel-titanium instruments: ProTaper versus Race. Part 2. Cleaning effectiveness and shaping ability in severely curved root canals of extracted teeth. Int Endod J 2004; 37(4):239-48.

51. Gagliardi J, Versiani MA, de Sousa-Neto MD, Plazas-Garzon A, Basrani B. Evaluation of the shaping characteristics of Pro Taper Gold, Pro Taper Next, and ProTaper Universal in curved canals. J Endod 2015; 41(10):1718-24.

52. Peters OA, Arias A, Paqué F. A micro-computed tomography assessment of root canal preparation with a novel instrument, TRUShape, in mesial roots of mandibular molars. J Endod 2015; 41:1545-50.

53. Amaral ROJF, Leonardi DP, Gabardo MCL, Coelho BS, Oliveira KV, Baratto-Filho F. Influence of cervical and apical enlargement associated with the WaveOne system on the transportation and centralization of endodontic preparations. J Endod 2016; 42:626-31.

\section{Endereço para correspondência:}

Caroline Felipe Magalhães Girelli

Rua Prudente de Morais, no 675 , apto 302, Centro

CEP 35020-460, Governador Valadares, Minas Gerais,

Brasil

Telefones: (33) 98860-2937, (33) 98822-2937

E-mail: carolfmagalhaes3@hotmail.com 